IASSNS-AST 97/63

UWThPh-1997-43

DFTT $67 / 97$

hep-ph/9711311

\title{
Neutrino mixing from neutrino oscillation data
}

\author{
S.M. Bilenky \\ Joint Institute for Nuclear Research, Dubna, Russia, and \\ Institute for Advanced Study, Princeton, N.J. 08540 \\ C. Giunti \\ INFN, Sezione di Torino, and Dipartimento di Fisica Teorica, Università di Torino, \\ Via P. Giuria 1, I-10125 Torino, Italy \\ W. Grimus \\ Institute for Theoretical Physics, University of Vienna, \\ Boltzmanngasse 5, A-1090 Vienna, Austria
}

\begin{abstract}
We show that the existing neutrino oscillation data are in favour of the schemes with mixing of four massive neutrinos and that only two of these schemes, with two pairs of neutrinos with close masses separated by a gap of about $1 \mathrm{eV}$, are compatible with all data. Possible implications of these schemes for future experiments are discussed.

Talk presented by S.M. Bilenky at the Erice School on Nuclear Physics, $19^{\text {th }}$ course "Neutrinos in Astro, Particle and Nuclear Physics", 16-24 September 1997.
\end{abstract}




\section{INTRODUCTION}

The problem of neutrino masses and mixing is the key problem of today's neutrino physics. The investigation of this problem is one of the major ways to search for new physics beyond the standard model. There exist three indications in favour of non-zero neutrino masses and neutrino mixing at present. The first indication comes from solar neutrino experiments [1]. The observed deficit of solar $\nu_{e}$ 's can be explained by neutrino mixing with $\Delta m^{2} \sim 10^{-5} \mathrm{eV}^{2}$ in the case of MSW transitions or with $\Delta m^{2} \sim 10^{-10} \mathrm{eV}^{2}$ in the case of vacuum oscillations $\left(\Delta m^{2}\right.$ is the relevant neutrino mass-squared difference). The second indication in favour of neutrino mixing comes from the observed deficit of atmospheric $\nu_{\mu}$ [2,:3]. From the data of the Kamiokande and Super-Kamiokande experiments [2] it follows that a second scale $\Delta m^{2} \sim 10^{-3}-10^{-2} \mathrm{eV}^{2}$ exists. The third indication was obtained in the LSND experiment [A]. The data of this experiment are in favour of the existence of a third

scale $\Delta m^{2} \sim 1 \mathrm{eV}^{2}$. There exist also data of numerous reactor and accelerator short-baseline (SBL) experiments in which no indications in favour of neutrino mixing were obtained (see [5]).

Which information about the neutrino mass spectrum and neutrino mixing and what implications for future experiments can be inferred from the existing data? We will discuss here these questions in the framework of a general phenomenological approach that takes into account only the unitarity of the mixing matrix [6]10].

We will start with a few general remarks about neutrino masses and mixing. There are two possibilities for massive neutrinos (see [11,12]):

1. Massive neutrinos can be Dirac particles like all other fundamental fermions. In this case the total lepton number $L=L_{e}+L_{\mu}+L_{\tau}$ is conserved and, in the simplest case, the number of massive neutrinos is equal to the number of lepton flavours. For neutrino mixing we have

$$
\nu_{\alpha L}=\sum_{i=1}^{3} U_{\alpha i} \nu_{i L} \quad(\alpha=e, \mu, \tau)
$$

where $U$ is the unitary mixing matrix and $\nu_{i}$ is the field of neutrinos with mass $m_{i}$.

2. Massive neutrinos can be truly neutral Majorana particles. In this case the fields of neutrinos with a definite mass, $\chi_{i}$, satisfy the Majorana condition

$$
\chi_{i}^{c}=C \bar{\chi}_{i}^{T}=\chi_{i}
$$

and there are no conserved lepton numbers ( $C$ is the charge conjugation matrix). The number of massive Majorana neutrinos is equal to three if only the left-handed neutrino fields, which enter in the interaction, enter in the neutrino mass term. In general lefthanded and right-handed neutrino fields can enter in the neutrino mass term. In this case the number $n$ of Majorana neutrinos is larger than three and for the mixing we have

$$
\nu_{\alpha L}=\sum_{i=1}^{n} U_{\alpha i} \chi_{i L}, \quad\left(\nu_{a R}\right)^{c}=\sum_{i=1}^{n} U_{a i} \chi_{i L},
$$


where $\nu_{a R}$ is a right-handed (sterile) field. Let us stress that LEP experiments on the measurement of invisible width of Z-boson, that proved that the number of flavour neutrinos is equal to three, do not exclude the possibility of existence of more than three massive light neutrinos (the right-handed fields do not enter in the standard neutral current).

Dirac neutrino masses can be generated by the standard mechanism with a Higgs doublet if the right-handed fields $\nu_{a R}$ enter in the Lagrangian and are singlets. Majorana neutrino masses can be generated only in the framework of theories in which lepton numbers are not conserved. The most popular mechanism of generation of Majorana neutrino masses is the see-saw mechanism [13]. This mechanism is based on the assumption that the lepton number is violated by the right-handed Majorana mass term at a scale $M$ that is much larger than the scales of the masses of leptons and quarks (usually $M \simeq M_{\mathrm{GUT}}$ ). In the standard see-saw case, for the neutrino masses we have

$$
m_{i} \simeq \frac{\left(m_{F i}\right)^{2}}{M} .
$$

where $m_{F i}$ is the mass of the lepton or up-quark in the $i^{\text {th }}$-generation. Thus, if the neutrino masses are of see-saw origin, there are three massive neutrinos with masses that satisfy the hierarchy relation

$$
m_{1} \ll m_{2} \ll m_{3} .
$$

\section{THREE MASSIVE NEUTRINOS}

We will start with the case of three massive neutrinos [14, 6, 7] and we will assume that there is a hierarchy of neutrino masses and $\Delta m_{21}^{2} \equiv m_{2}^{2}-m_{1}^{2}$ is relevant for the suppression of the flux of solar $\nu_{e}$ 's. Let us consider SBL neutrino oscillations in such a scheme. Taking into account that $\frac{\Delta m_{21}^{2} L}{2 p} \ll 1$ ( $L$ is the source-detector distance and $p$ is the neutrino momentum), for the probability of $\nu_{\alpha} \rightarrow \nu_{\beta}$ transition we have

$$
P_{\nu_{\alpha} \rightarrow \nu_{\beta}}=\left|\delta_{\alpha \beta}+U_{\beta 3} U_{\alpha 3}^{*}\left(e^{-i \frac{\Delta m^{2} L}{2 p}}-1\right)\right|^{2},
$$

with $\Delta m^{2} \equiv \Delta m_{31}^{2} \equiv m_{3}^{2}-m_{1}^{2}$. From this expression we easily find

$$
\begin{aligned}
& P_{\nu_{\alpha} \rightarrow \nu_{\beta}}=\frac{1}{2} A_{\beta ; \alpha}\left(1-\cos \frac{\Delta m^{2} L}{2 p}\right), \quad \text { for } \quad \beta \neq \alpha, \\
& P_{\nu_{\alpha} \rightarrow \nu_{\alpha}}=1-\frac{1}{2} B_{\alpha ; \alpha}\left(1-\cos \frac{\Delta m^{2} L}{2 p}\right) .
\end{aligned}
$$

with the oscillation amplitudes $A_{\beta ; \alpha}$ and $B_{\alpha ; \alpha}$ given by

$$
\begin{aligned}
& A_{\beta ; \alpha}=4\left|U_{\beta 3}\right|^{2}\left|U_{\alpha 3}\right|^{2}, \\
& B_{\alpha ; \alpha}=\sum_{\beta \neq \alpha} A_{\beta ; \alpha}=4\left|U_{\alpha 3}\right|^{2}\left(1-\left|U_{\alpha 3}\right|^{2}\right) .
\end{aligned}
$$


In the case of a hierarchy of neutrino masses, only one oscillation length characterizes neutrino oscillations in SBL experiments. It is obvious that the dependence of the transition probabilities on the quantity $\Delta m^{2} L / 2 p$ has the same form as in the two-neutrino case. Let us stress, however, that the expressions (2.2) and (2.3) are general and describe transitions between all three flavour neutrinos. Notice also that in the case of a hierarchy of neutrino masses the CP phase does not enter in the expressions for the transition probabilities. As a result we have

$$
P_{\nu_{\alpha} \rightarrow \nu_{\beta}}=P_{\bar{\nu}_{\alpha} \rightarrow \bar{\nu}_{\beta}}
$$

With the help of Eqs.(2.3) and (2.5), one can obtain bounds on the mixing parameters $\left|U_{e 3}\right|^{2}$ and $\left|U_{\mu 3}\right|^{2}$ from exclusive plots that were found from the data of SBL reactor and accelerator disappearance experiments (because of unitarity of the mixing matrix $\left|U_{\tau 3}\right|^{2}=$ $\left.1-\left|U_{e 3}\right|^{2}-\left|U_{\mu 3}\right|^{2}\right)$.

We will consider the range

$$
10^{-1} \mathrm{eV}^{2} \leq \Delta m^{2} \leq 10^{3} \mathrm{eV}^{2}
$$

From the exclusion curves of SBL disappearance experiments, at any fixed value of $\Delta m^{2}$ we obtain the upper bounds $B_{\alpha ; \alpha} \leq B_{\alpha ; \alpha}^{0}$ for $\alpha=e, \mu$. From Eq.(2.5), for the mixing parameters $\left|U_{\alpha 3}\right|^{2}$ we have

$$
\left|U_{\alpha 3}\right|^{2} \leq a_{\alpha}^{0} \quad \text { or } \quad\left|U_{\alpha 3}\right|^{2} \geq 1-a_{\alpha}^{0}, \quad \text { with } \quad a_{\alpha}^{0}=\frac{1}{2}\left(1-\sqrt{1-B_{\alpha ; \alpha}^{0}}\right) .
$$

We have obtained the values of $a_{e}^{0}$ and $a_{\mu}^{0}$ from the exclusion plots of the Bugey reactor experiment [15] and the CDHS and CCFR accelerator experiments [16] (see Fig.1 of [0]). In the range (2.7) we have $a_{e}^{0} \lesssim 4 \times 10^{-2}$ and $a_{\mu}^{0} \lesssim 2 \times 10^{-1}$ (for $\Delta m^{2} \gtrsim 0.3 \mathrm{eV}^{2}$ ). Thus, from the results of disappearance experiments it follows that the mixing parameters $\left|U_{e 3}\right|^{2}$ and $\left|U_{\mu 3}\right|^{2}$ can be either small or large (close to one).

Now let us take into account the results of solar neutrino experiments. For the probability of solar neutrinos to survive in the case of neutrino mass hierarchy we have the following lower bound:

$$
P_{\nu_{e} \rightarrow \nu_{e}}^{\operatorname{sun}} \geq\left|U_{e 3}\right|^{4}
$$

If $\left|U_{e 3}\right|^{2} \geq 1-a_{e}^{0}$, from (2.9) it follows that at all solar neutrino energies $P_{\nu_{e} \rightarrow \nu_{e}}^{\text {sun }} \gtrsim 0.92$. This is not compatible with the results of solar neutrino experiments. Thus, the mixing parameter $\left|U_{e 3}\right|^{2}$ must be small: $\left|U_{e 3}\right|^{2} \leq a_{e}^{0}$.

We come to the conclusion that from the results of SBL inclusive experiments and solar neutrino experiments it follows that in the case of three massive neutrinos with a hierarchy of masses only two schemes of mixing are possible:

$$
\text { I. }\left\{\begin{array} { l } 
{ | U _ { e 3 } | ^ { 2 } \leq a _ { e } ^ { 0 } , } \\
{ | U _ { \mu 3 } | ^ { 2 } \leq a _ { \mu } ^ { 0 } , }
\end{array} \quad \text { II. } \left\{\begin{array}{l}
\left|U_{e 3}\right|^{2} \leq a_{e}^{0}, \\
\left|U_{\mu 3}\right|^{2} \geq 1-a_{\mu}^{0} .
\end{array}\right.\right.
$$

Let us consider $\nu_{\mu} \leftrightarrows \nu_{e}$ oscillations in the case of scheme I. From Eqs.(2.4) and (2.10), for the oscillation amplitude we have 


$$
A_{e ; \mu} \leq 4\left|U_{e 3}\right|^{2}\left|U_{\mu 3}\right|^{2} \leq 4 a_{e}^{0} a_{\mu}^{0},
$$

i.e. the upper bound for the amplitude $A_{e ; \mu}$ is quadratic in the small quantities $a_{e}^{0}, a_{\mu}^{0}$; thus, in the case of scheme I $\nu_{\mu} \leftrightarrows \nu_{e}$ oscillations are strongly suppressed.

Let us compare now the upper bound (2.11) with the results of the LSND experiment in which $\nu_{\mu} \leftrightarrows \nu_{e}$ oscillations were observed. In Fig.1 the shadowed region in the $A_{e ; \mu}-\Delta m^{2}$ plane is the region allowed by the results of the LSND experiment. The regions excluded by the Bugey experiment [15] and by the BNL E734, BNL E776 and CCFR experiments [17 are also shown. The upper bound (2.11) is presented by the curve passing through the circles. As it is seen from Fig.1, the upper bound (2.11) is not compatible with the results of the LSND experiment if the results of other oscillation experiments are taken into account. Thus, the scheme I with a hierarchy of masses and couplings, similar to the hierarchy that takes place in the quark sector, is not favoured by the results of SBL experiments.

In the case of scheme II, the upper bound of the amplitude $A_{e ; \mu}$ is linear in the small quantity $a_{e}^{0}: A_{e ; \mu} \leq 4 a_{e}^{0}$. This upper bound is compatible with the LSND data. Note that, if scheme II is realized in nature, $\left|\nu_{\mu}\right\rangle \simeq\left|\nu_{3}\right\rangle$ and the vectors $\left|\nu_{e}\right\rangle$ and $\left|\nu_{\tau}\right\rangle$ are superpositions of $\left|\nu_{1}\right\rangle$ and $\left|\nu_{2}\right\rangle$. For the neutrino masses we have $m_{\nu_{\mu}} \simeq m_{3}, m_{\nu_{e}}, m_{\nu_{\tau}} \ll m_{3}$.

Up to now we did not consider atmospheric neutrinos. In the framework of the scheme with three massive neutrinos and a neutrino mass hierarchy there are only two possibilities to take into account the atmospheric neutrino anomaly:

1. To assume that $\Delta m_{21}^{2}$ is relevant for the suppression of solar $\nu_{e}$ 's and for the atmospheric neutrino anomaly 18 .

2. To assume that $\Delta m_{31}^{2}$ is relevant for the LSND anomaly and for the atmospheric neutrino anomaly [19].

In first case, the average survival probability of solar $\nu_{e}$ 's is constant (this is disfavoured by the data of solar neutrino experiments [20,21]) and the parameters $\left|U_{e 3}\right|^{2},\left|U_{\mu 3}\right|^{2}$ satisfy inequalities $\left|U_{e 3}\right|^{2} \leq a_{e}^{0}$ and $\left|U_{\mu 3}\right|^{2} \leq a_{\mu}^{0}$ (that are not compatible with the LSND result, as we have discussed above).

In the second case it is not possible to explain the angular dependence of the double ratio of muon and electron events that was observed by the Kamiokande and Super-Kamiokande experiments [2].

All the existing indications in favour of neutrino mixing will be checked by several experiments that now are under preparation. If for the time being we accept them, we come to the necessity of consideration of schemes with four massive neutrinos, that include the three flavour neutrinos $\nu_{e}, \nu_{\mu}, \nu_{\tau}$ and a sterile neutrino [22,8 10].

\section{FOUR MASSIVE NEUTRINOS}

There are six possible types of mass spectra with four neutrinos that can accommodate three different scales of $\Delta m^{2}$. Let us start with the case of a hierarchy of neutrino masses $m_{1} \ll m_{2} \ll m_{3} \ll m_{4}$, assuming that $\Delta m_{21}^{2}$ is relevant for the suppression of solar $\nu_{e}$ 's and $\Delta m_{31}^{2}$ is relevant for the atmospheric neutrino anomaly. The SBL transition probabilities are 
given in this case by the expressions (2.2)-(2.5) with $\left|U_{\alpha 3}\right|^{2} \rightarrow\left|U_{\alpha 4}\right|^{2}$ and $\Delta m^{2} \equiv \Delta m_{41}^{2} \equiv$ $m_{4}^{2}-m_{1}^{2}$. From SBL inclusive data in the range (2.7) of $\Delta m^{2}$, we have

$$
\left|U_{\alpha 4}\right|^{2} \leq a_{\alpha}^{0} \quad \text { or } \quad\left|U_{\alpha 4}\right|^{2} \geq 1-a_{\alpha}^{0}, \quad \text { for } \quad \alpha=e, \mu .
$$

For the survival probability of the atmospheric $\nu_{\mu}$ 's in the scheme under consideration we have the lower bound [9]

$$
P_{\nu_{\mu} \rightarrow \nu_{\mu}}^{\mathrm{atm}} \geq\left|U_{\mu 4}\right|^{4} .
$$

Now, from Eq.(2.9) with $\left|U_{e 3}\right|^{2} \rightarrow\left|U_{e 4}\right|^{2}$ and from Eq.(3.2) it follows that large values of the mixing parameters $\left|U_{e 4}\right|^{2}$ and $\left|U_{\mu 4}\right|^{2}$ are not compatible with solar and atmospheric neutrino data. We come to the conclusion that both mixing parameters $\left|U_{e 4}\right|^{2}$ and $\left|U_{\mu 4}\right|^{2}$ are small: $\left|U_{e 4}\right|^{2} \leq a_{e}^{0}$ and $\left|U_{\mu 4}\right|^{2} \leq a_{\mu}^{0}$. As in the case of scheme I for three neutrinos, in the scheme under consideration the SBL amplitude $A_{e ; \mu}$ is constrained by the upper bound (2.11) (with $\left|U_{\alpha 3}\right|^{2} \rightarrow\left|U_{\alpha 4}\right|^{2}$ ), which is not compatible with the LSND allowed region (see Fig.1). Thus, a mass hierarchy of four neutrinos is not favoured by the existing data. The same conclusion can be drawn for all four-neutrino mass spectra with one neutrino mass separated from the group of three close masses by the "LSND gap" $(\sim 1 \mathrm{eV})$.

Let us consider now the two possible neutrino mass spectra

$$
\underbrace{\overbrace{m_{1}<m_{2}}^{\text {atm }} \ll \overbrace{m_{3}<m_{4}}^{\text {sun }}}_{\text {LSND }},
$$

$$
\underbrace{\overbrace{m_{1}<m_{2}}^{\text {sun }} \ll \overbrace{m_{3}<m_{4}}^{\text {atm }}}_{\text {LSND }},
$$

with two groups of close masses separated by a $\sim 1 \mathrm{eV}$ gap. In the case of such neutrino mass spectra, the SBL transition probabilities are given by the expressions (2.2) and (2.3) and the oscillation amplitudes are given by

$$
A_{\beta ; \alpha}=4\left|\sum_{i} U_{\beta i} U_{\alpha i}^{*}\right|^{2}, \quad B_{\alpha ; \alpha}=4\left(\sum_{i}\left|U_{\alpha i}\right|^{2}\right)\left(1-\sum_{i}\left|U_{\alpha i}\right|^{2}\right) .
$$

where the index $i$ runs over 1,2 or 3,4 . From the exclusion plots of the Bugey, CDHS and CCFR disappearance experiments we have

$$
\sum_{i}\left|U_{\alpha i}\right|^{2} \leq a_{\alpha}^{0} \quad \text { or } \quad \sum_{i}\left|U_{\alpha i}\right|^{2} \geq 1-a_{\alpha}^{0}, \quad \text { for } \quad \alpha=e, \mu .
$$

If we take into account the results of solar and atmospheric neutrino experiments, in the case of scheme A we have

$$
\sum_{i=1,2}\left|U_{e i}\right|^{2} \leq a_{e}^{0} \quad \text { and } \quad \sum_{i=3,4}\left|U_{\mu i}\right|^{2} \leq a_{\mu}^{0} .
$$

The corresponding inequalities in the scheme B can be obtained from Eq.(3.6) with the change $1,2 \leftrightarrows 3,4$

Now, for the amplitude of $\nu_{\mu} \leftrightarrows \nu_{e}$ oscillations, from Eqs.(3.4) and (3.6), in both schemes we have the upper bound 


$$
A_{e ; \mu}=4\left|\sum_{i} U_{\mu i} U_{e i}^{*}\right|^{2} \leq 4\left(\sum_{i}\left|U_{\mu i}\right|^{2}\right)\left(\sum_{i}\left|U_{e i}\right|^{2}\right) \leq 4 \operatorname{Min}\left[a_{e}^{0}, a_{\mu}^{0}\right],
$$

that is compatible with the LSND result. Thus, schemes A and B can accommodate all neutrino oscillation data.

The schemes $\mathrm{A}$ and $\mathrm{B}$ give different predictions for the neutrino mass $m\left({ }^{3} \mathrm{H}\right)$ measured in ${ }^{3} \mathrm{H}$-decay experiments, and for the effective Majorana mass $\langle m\rangle=\sum_{i=1}^{4} U_{e i}^{2} m_{i}$ that enters into matrix element of neutrinoless double-beta decay. In the scheme $\mathrm{A}$ we have

$$
m\left({ }^{3} \mathrm{H}\right) \simeq m_{4}
$$

In the case of scheme $B$, the contribution to the beta-spectrum of the term that includes the heaviest masses $m_{3} \simeq m_{4}$ is suppressed by the factor $\sum_{i=3,4}\left|U_{e i}\right|^{2} \leq a_{e}^{0} \lesssim 4 \times 10^{-2}$.

For the the effective Majorana mass in neutrinoless double-beta decay, in the schemes $\mathrm{A}$ and $\mathrm{B}$ we have

$$
\text { (A) } \quad|\langle m\rangle| \leq \sum_{i=3,4}\left|U_{e i}\right|^{2} m_{4} \leq m_{4}, \quad \text { (B) } \quad|\langle m\rangle| \leq a_{e}^{0} m_{4} \ll m_{4} .
$$

Thus, if the scheme $\mathrm{A}$ is realized in nature, the ${ }^{3} \mathrm{H}$-decay experiments on the direct measurement of neutrino mass and the experiments on the search for neutrinoless doublebeta decay can see the effect of the "LSND neutrino mass".

Finally, we will consider neutrino oscillations in long-baseline (LBL) experiments in the framework of the schemes A and B. We will show that the data SBL experiments imply rather strong constrains on the LBL probabilities of $\bar{\nu}_{e} \rightarrow \bar{\nu}_{e}$ and $\nu_{\mu} \rightarrow \nu_{e}$ transitions [10]. In the scheme A, for the probability of LBL $\nu_{\alpha} \rightarrow \nu_{\beta}$ transitions we have the following expression:

$$
P_{\nu_{\alpha} \rightarrow \nu_{\beta}}^{(\mathrm{LBL}, \mathrm{A})}=\left|U_{\beta 1} U_{\alpha 1}^{*}+U_{\beta 2} U_{\alpha 2}^{*} \exp \left(-i \frac{\Delta m_{21}^{2} L}{2 p}\right)\right|^{2}+\left|\sum_{k=3,4} U_{\beta k} U_{\alpha k}^{*}\right|^{2}
$$

The probability of the $\nu_{\alpha} \rightarrow \nu_{\beta}$ transitions in scheme B can be obtained from Eq.(3.10) with the change $1,2 \leftrightarrows 3,4$. Let us notice also that the probability of LBL $\bar{\nu}_{\alpha} \rightarrow \bar{\nu}_{\beta}$ transition can be obtained from Eq.(3.10) with the change $U_{\alpha k} \rightarrow U_{\alpha k}^{*}$.

Two reactor experiments, CHOOZ [23] and Palo Verde [24], are the first long-baseline experiments. From Eq.(3.10), for the probabilities of $\bar{\nu}_{e} \rightarrow \bar{\nu}_{e}$ transition in the schemes A and $\mathrm{B}$ we have the following lower bounds:

$$
P_{\bar{\nu}_{e} \rightarrow \bar{\nu}_{e}}^{(\mathrm{LBL}, \mathrm{A})} \geq\left(\sum_{i=3,4}\left|U_{e i}\right|^{2}\right)^{2}
$$

$$
P_{\bar{\nu}_{e} \rightarrow \bar{\nu}_{e}}^{(\mathrm{LBL})} \geq\left(\sum_{i=1,2}\left|U_{e i}\right|^{2}\right)^{2}
$$

Now, taking into account the unitarity of the mixing matrix, we can conclude that the quantities in the right-hand sides of the two inequalities (3.11) are large. Thus, on the basis of the existing neutrino oscillation data, we expect that the probability $P_{\bar{\nu}_{e} \rightarrow \bar{\nu}_{e}}^{(\mathrm{LBL})}$ is close to one. Indeed, from Eq.(3.11), for both schemes we have 


$$
P_{\bar{\nu}_{e} \rightarrow \bar{\nu}_{e}}^{(\mathrm{LBL})} \geq\left(1-a_{e}^{0}\right)^{2}
$$

For the transition probability of $\bar{\nu}_{e}$ into any other state, from Eq.(3.12) we have the upper bound

$$
1-P_{\bar{\nu}_{e} \rightarrow \bar{\nu}_{e}}^{(\mathrm{LBL})}=\sum_{\alpha \neq e} P_{\bar{\nu}_{e} \rightarrow \bar{\nu}_{\alpha}}^{(\mathrm{LBL})} \leq a_{e}^{0}\left(2-a_{e}^{0}\right)
$$

The value of $a_{e}^{0}$ depends on the SBL parameter $\Delta m^{2}$. In Fig.2 we have drawn the curve corresponding to the upper bound (3.13) for $\Delta m^{2}$ in the interval (2.7). The minimum values

of the probability $\sum_{\alpha \neq e} P_{\bar{\nu}_{e} \rightarrow \bar{\nu}_{\alpha}}^{(\mathrm{LBL})}$ that is planned to be reached in the CHOOZ and Palo Verde experiments are also shown. The shadowed region in Fig.2 is the region that is allowed (at 90\% CL) by the data of the LSND experiment and of the other SBL experiments. Thus, as it is seen from Fig.2, in the framework of the schemes A and B, the existing data put rather severe constraints on the LBL transition probability of $\bar{\nu}_{e}$ into any other state.

Taking into account the unitarity of the mixing matrix and the CPT-theorem, it easy to see that the LBL probability of $\nu_{\mu} \rightarrow \nu_{e}$ transitions is also strongly suppressed. Indeed, we have

$$
P_{\nu_{\mu} \rightarrow \nu_{e}}^{(\mathrm{LBL})}=P_{\bar{\nu}_{e} \rightarrow \bar{\nu}_{\mu}}^{(\mathrm{LBL})} \leq a_{e}^{0}\left(2-a_{e}^{0}\right)
$$

Another upper bound on the LBL probability of $\nu_{\mu} \rightarrow \nu_{e}$ transitions can be obtained from Eqs.(3.6) and (3.10). For both models we have

$$
P_{\nu_{\mu} \rightarrow \nu_{e}}^{(\mathrm{LBL})} \leq a_{e}^{0}+\frac{1}{4} A_{e ; \mu} .
$$

The upper bound for the LBL probability of $\nu_{\mu} \rightarrow \nu_{e}$ transitions, obtained with the help of Eqs.(3.14) and (3.15), is shown in Fig.3 by the short-dashed curve. The solid line presents the corresponding bound with matter corrections for the $\mathrm{K} 2 \mathrm{~K}$ experiment [25]. The dash-dotted vertical line presents the expected minimal value of the probability $P_{\nu_{\mu} \rightarrow \nu_{e}}^{(\mathrm{LBL})}$ that will be reached in the $\mathrm{K} 2 \mathrm{~K}$ experiment. Notice that at all the considered values of $\Delta m^{2}$ this probability is larger than the upper bound with matter corrections. The solid line in Fig. 14 shows the bound corresponding to Eqs.(3.14) and (3.15) with matter corrections for the MINOS [26] and ICARUS [27] experiments, whose expected sensitivities are represented, respectively, by the dash-dotted and dash-dot-dotted lines. One can see that these sensitivities are sufficient to explore the region allowed by the results of the LSND and the other SBL experiments.

\section{CONCLUSIONS}

The existing indications in favour of neutrino oscillations (coming from solar, atmospheric and the LSND experiments) require schemes with mixing of four massive neutrinos. We have shown that only two possible schemes with two pairs of neutrinos with close masses separated by a gap of about $1 \mathrm{eV}$ can accommodate all the existing neutrino oscillation data. We have discussed the possible implications of these schemes for the experiments 
on the direct measurement of the electron neutrino mass, on the search for neutrinoless double-beta decay and for long-baseline neutrino experiments $\rrbracket$.

\section{ACKNOWLEDGMENTS}

S.M.B. would like to acknowledge support from the Dyson Visiting Professor Funds at the Institute for Advanced Study.

1 After we finished this paper the first results of the CHOOZ experiment appeared [28]. No indications in favor of $\bar{\nu}_{e} \rightarrow \bar{\nu}_{e}$ transitions were found in this experiment. The upper bound for the probability $1-P_{\bar{\nu}_{e} \rightarrow \bar{\nu}_{e}}^{(\mathrm{LBL})}$ found in the CHOOZ experiment is in agreement with the bound obtained in [10] and presented in Fig.2. 


\section{REFERENCES}

[1] B.T. Cleveland et al., Nucl. Phys. B (P.S.) 38, 47 (1995); K.S. Hirata et al., Phys. Rev. D 44, 2241 (1991); GALLEX Coll., Phys. Lett. B 388, 384 (1996); SAGE Coll., Phys. Rev. Lett. 77, 4708 (1996); K. Inoue, Talk presented at TAUP97, September 7-11, 1997, Laboratori Nazionali del Gran Sasso, Assergi (Italy).

[2] Y. Fukuda et al., Phys. Lett. B 335, 237 (1994); K. Martens, Talk presented at the International Europhysics Conference on High Energy Physics, 19-26 August 1997, Jerusalem, Israel (http://www.cern.ch/hep97/abstract/tpa10.htm).

[3] R. Becker-Szendy et al., Nucl. Phys. B (P.S.) 38, 331 (1995); W.W.M. Allison et al., Phys. Lett. B 391, 491 (1997).

[4] C. Athanassopoulos et al., Phys. Rev. Lett. 77, 3082 (1996).

[5] F. Boehm, Nucl. Phys. B (Proc. Suppl.) 48, 148 (1996); F. Vannucci, ibid., 154 (1996).

[6] S.M. Bilenky, A. Bottino, C. Giunti and C.W. Kim, Phys. Lett. B 356, 273 (1995).

[7] S.M. Bilenky, A. Bottino, C. Giunti and C.W. Kim, Phys. Rev. D 54, 1881 (1996).

[8] S.M. Bilenky, C. Giunti, C.W. Kim and S.T. Petcov, Phys. Rev. D 54, 4432 (1996).

[9] S.M. Bilenky, C. Giunti and W. Grimus, preprint hep-ph/9607372, to appear in Z. Phys. C.

[10] S.M. Bilenky, C. Giunti and W. Grimus, preprint hep-ph/9710209, to appear in Phys. Rev. D.

[11] S.M. Bilenky and B. Pontecorvo, Phys. Rep. 41, 225 (1978).

[12] S.M. Bilenky and S.T. Petcov, Rev. Mod. Phys. 59, 671 (1987).

[13] M. Gell-Mann, P. Ramond, and R. Slansky, in Supergravity, North Holland, Amsterdam, 1979, p.315; T. Yanagida, Proc. of the Workshop on Unified Theory and the Baryon Number of the Universe, KEK, Japan, 1979; S. Weinberg, Phys. Rev. Lett. 43, 1566 (1979).

[14] A. De Rujula et al., Nucl. Phys. B 168, 54 (1980); V. Barger and K. Whisnant, Phys. Lett. B 209, 365 (1988); S.M. Bilenky et al., ibid. 276, 223 (1992); K.S. Babu et al., ibid. 359, 351 (1995). H. Minakata, ibid. 356, 61 (1995); Phys. Rev. D 52, 6630 (1995); G.L. Fogli et al., ibid. 52, 5334 (1995); S. Goswami et al., Int. J. Mod. Phys. A 12, 781 (1997).

[15] B. Achkar et al., Nucl. Phys. B 434, 503 (1995).

[16] F. Dydak et al., Phys. Lett. B 134, 281 (1984); I.E. Stockdale et al., Phys. Rev. Lett. 52, 1384 (1984).

[17] L.A.Ahrens et al., Phys. Rev. D 36, 702 (1987); L.Borodovsky et al., Phys. Rev. Lett. 68, 274 (1992); A.Romosan et al., ibid 78, 2912 (1997).

[18] A. Acker and S. Pakvasa, Phys. Lett. B 357, 209 (1997).

[19] C.Y. Cardall and G.M. Fuller, Phys. Rev. D 53, 4421 (1996); C.Y. Cardall, G.M. Fuller and D.B. Cline, preprint hep-ph/9706426.

[20] P.I. Krastev and S.T. Petcov, Phys. Lett. B 395, 69 (1997).

[21] G. Conforto, A. Marchionni, F. Martelli and F. Vetrano, preprint hep-ph/9708301.

[22] J.T. Peltoniemi and J.W.F. Valle, Nucl. Phys. B 406, 409 (1993); D.O. Caldwell and R.N. Mohapatra, Phys. Rev. D 48, 3259 (1993); Z. Berezhiani and R.N. Mohapatra, ibid 52, 6607 (1995); J.R. Primack et al., Phys. Rev. Lett. 74, 2160 (1995); E. Ma and P. Roy, Phys. Rev. D 52, R4780 (1995); R. Foot and R.R. Volkas, ibid 52, 6595 
(1995); E.J. Chun et al., Phys. Lett. B 357, 608 (1995); J.J. Gomez-Cadenas and M.C. Gonzalez-Garcia, Z. Phys. C 71, 443 (1996); S. Goswami, Phys. Rev. D 55, 2931 (1997); A.Yu. Smirnov and M. Tanimoto, ibid 55, 1665 (1997); E. Ma, Mod. Phys. Lett. A 11, 1893 (1996).

[23] R.I. Steinberg, Proc. of the $5^{\text {th }}$ International Workshop on Neutrino Telescopes, Venezia, March 1993; http://duphy4.physics.drexel.edu/chooz_pub/.

[24] F. Boehm et al., The Palo Verde experiment, 1996; http://www.cco.caltech.-

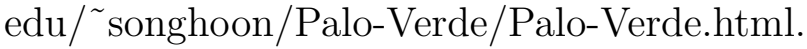

[25] Y. Suzuki, Talk presented at Neutrino 96, Helsinki, June 1996; http://pnahp.kek.jp/.

[26] MINOS Coll., NUMI-L-63, February 1995; http://www.hep.anl.gov/NDK/HyperText/numi.html.

[27] ICARUS Coll., LNGS-94/99-I, May 1994; http://www.aquila.infn.it/icarus/.

[28] M. Apollonio et al., preprint hep-ex/9711002. 


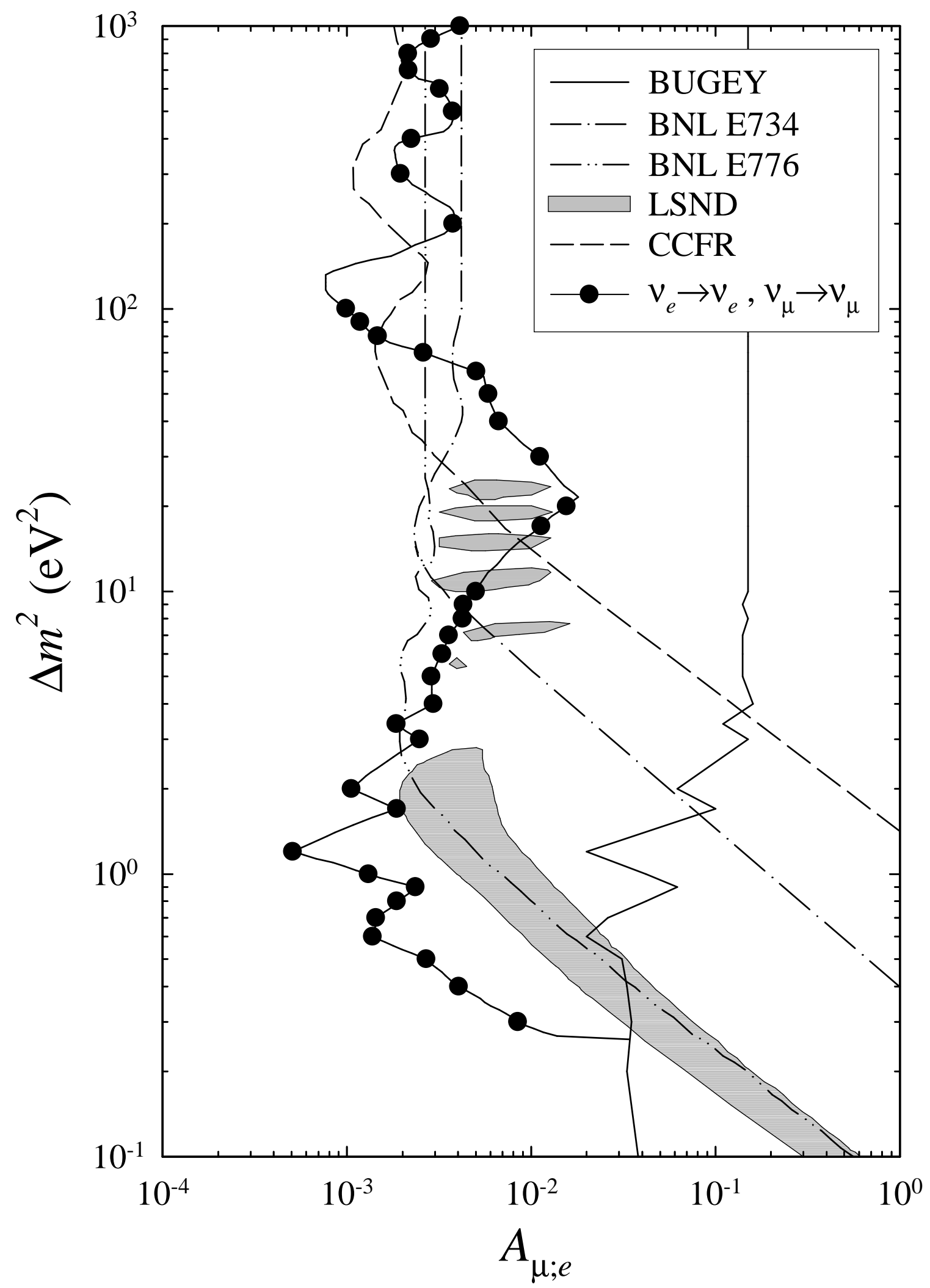

Figure 凹 


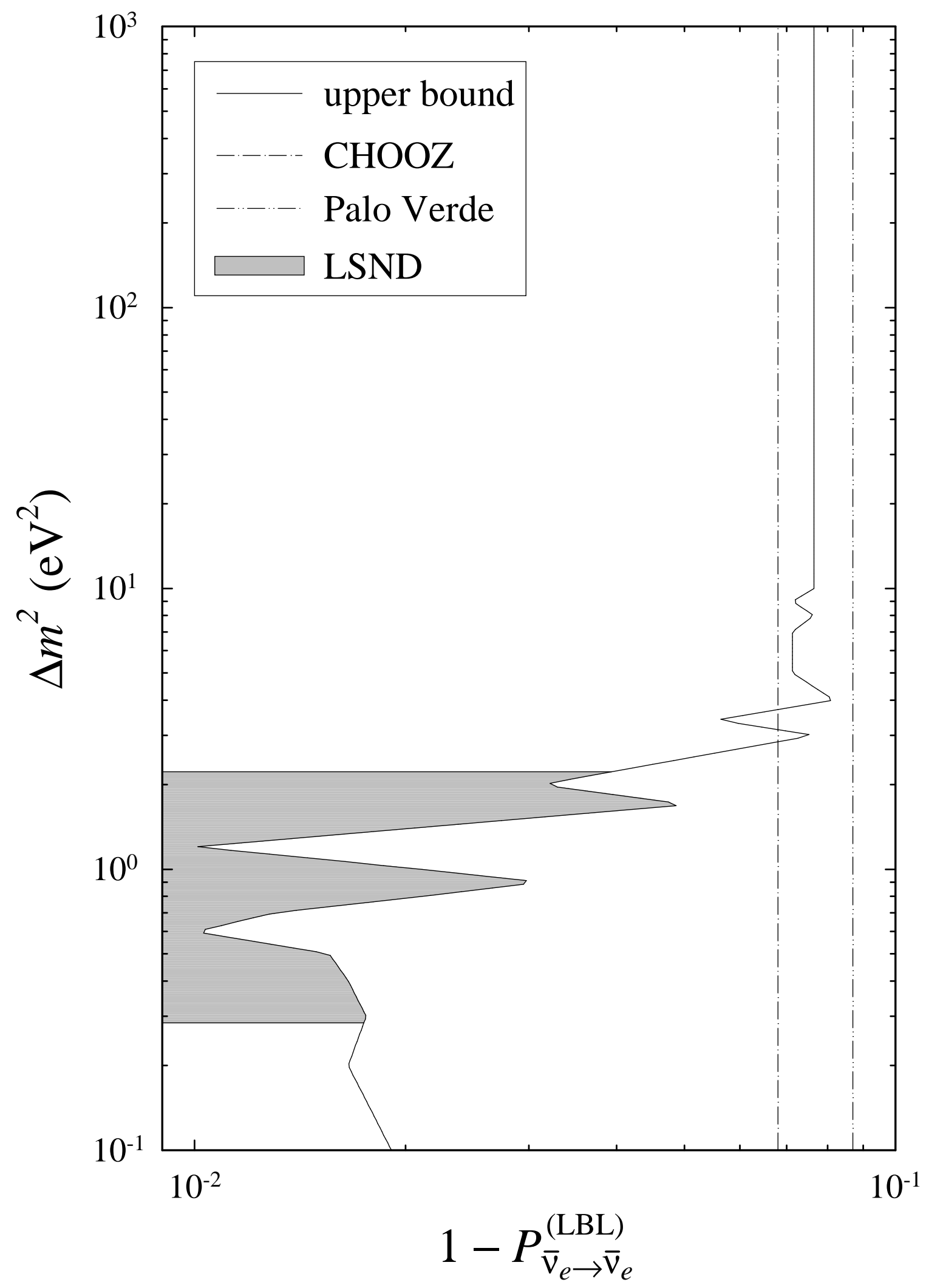

Figure [ 


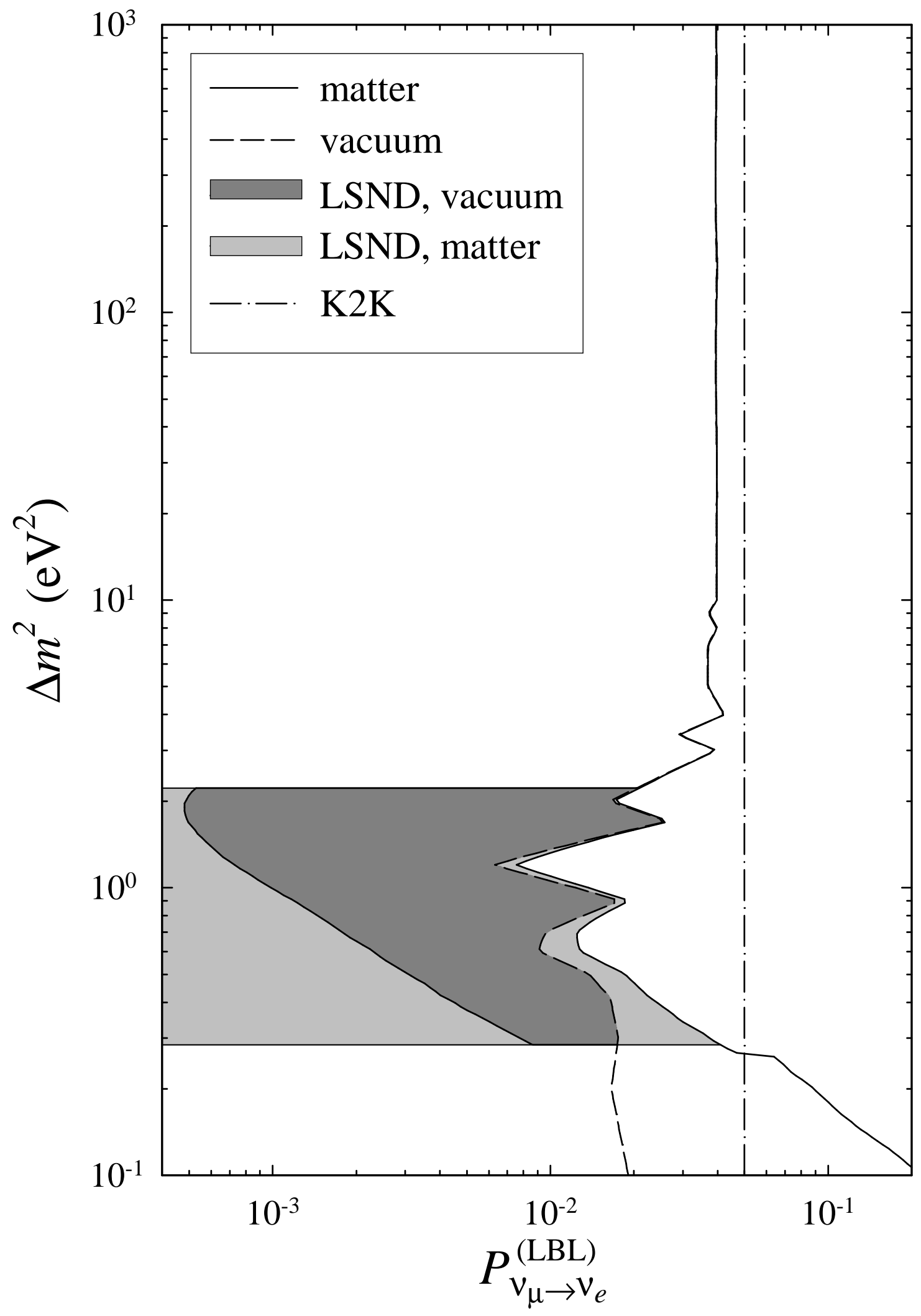

Figure 3 


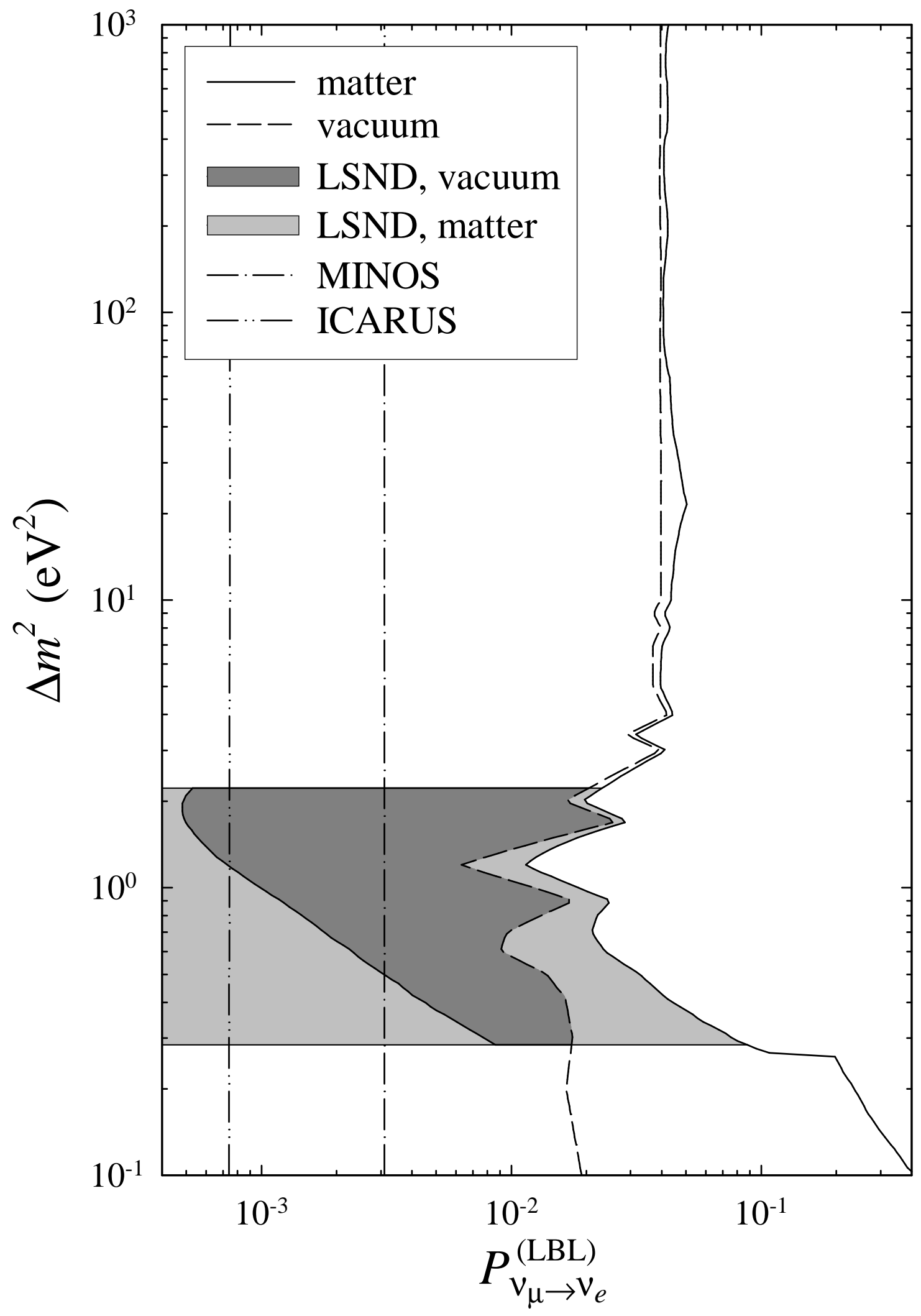

Figure 由 